Case Report

\title{
Acute Awake Fiberoptic Intubation in the ICU in a Patient with Limited Mouth Opening and Hypoxemic Acute Respiratory Failure
}

\author{
Kjartan E. Hannig $\mathbb{D}^{1},{ }^{1}$ Rasmus W. Hauritz $\mathbb{D}^{1},{ }^{1}$ Christian Jessen, ${ }^{2}$ and Anders M. Grejs ${ }^{3}$ \\ ${ }^{1}$ Department of Anesthesiology, Kolding Hospital, 6000 Kolding, Denmark \\ ${ }^{2}$ Department of Anesthesiology, Horsens Hospital, 8700 Horsens, Denmark \\ ${ }^{3}$ Department of Intensive Care Medicine, Aarhus University Hospital, 8200 Aarhus N, Denmark
}

Correspondence should be addressed to Kjartan E. Hannig; kjartanhannig@yahoo.com

Received 9 June 2019; Revised 31 July 2019; Accepted 19 August 2019; Published 23 October 2019

Academic Editor: Pavel Michalek

Copyright (c) 2019 Kjartan E. Hannig et al. This is an open access article distributed under the Creative Commons Attribution License, which permits unrestricted use, distribution, and reproduction in any medium, provided the original work is properly cited.

\begin{abstract}
The incidence and survival of patients with head-and-neck cancer have been on the increase for decades. Following surgery or radiation therapy, complications such as difficult airways may evolve. These difficult airways may be unique and not manageable with conventional intubation methods as well as video laryngoscopes. Acute awake fiberoptic intubation may be a feasible option also for urgent emergency airway management of known difficult airways. The "cannot intubate-cannot oxygenate" (CI-CO) situation has to be avoided at all costs, since emergency cricothyrotomy has a fail ratio of more than $50 \%$ when performed by an anesthesiologist.
\end{abstract}

\section{Introduction}

In Denmark, the incidence of head-and-neck cancer (HNC) has more than doubled since 1980 and the relative 5-year survival has risen from $49 \%$ to $72 \%$ [1]. Previous HNC surgery or radiation therapy is a strong predictor of a future difficult airway [2]. Of all patients undergoing an emergency cricothyrotomy due to a "cannot intubate-cannot oxygenate" (CI-CO) scenario, $75 \%$ went through HNC surgery at some point prior to the event [2]. Hence, it is likely that the proportion of patients with potentially difficult airways will increase in hospitals without ear-nose-throat (ENT) expertise, with acute symptoms requiring immediate treatment. Our hypothesis is that all hospitals managing patients in acute respiratory failure should not only have backup plans for unexpected difficult airways but also for the expected ones [3]. This optimally includes skills in awake fiberoptic intubation (FOI), which preserves spontaneous breathing and thus can prevent the risk of critical desaturation or a CI-CO situation [4].

\section{Case Presentation}

Written informed patient consent for publication was obtained. Approval from The Central Denmark Region Committee on Health Research Ethics was unwarranted.

A 71-year-old man (height $166 \mathrm{~cm}$, weight $60 \mathrm{~kg}$ ) with previous $\mathrm{HNC}$, presented in the emergency room due to fever and increasing dyspnea during the last 4 days.

On arrival, the patient was fully alert and oriented. Respiratory rate was 40 breaths $/ \mathrm{min}$, peripheral saturation was $80 \%$ with $10 \mathrm{~L} / \mathrm{min}$ oxygen delivered on a Hudson mask, blood pressure was $91 / 64 \mathrm{mmHg}$, heart rate was 110 beats $/ \mathrm{min}$, and temperature was $38.5^{\circ} \mathrm{C}$. The Hudson mask was replaced with a reservoir mask with an oxygen flow of $15 \mathrm{~L} / \mathrm{min}$. A computed tomography scan including intravenous contrast was performed, which showed bilateral pneumonia and excluded pulmonary embolism (Figure 1). The patient was transferred to the intensive care unit (ICU), where high-flow nasal oxygen (HFNO) was administered with a flow of $60 \mathrm{~L} / \mathrm{min}$ and a fraction of inspired oxygen $\left(\mathrm{FiO}_{2}\right)$ of $100 \%$. However, this was 


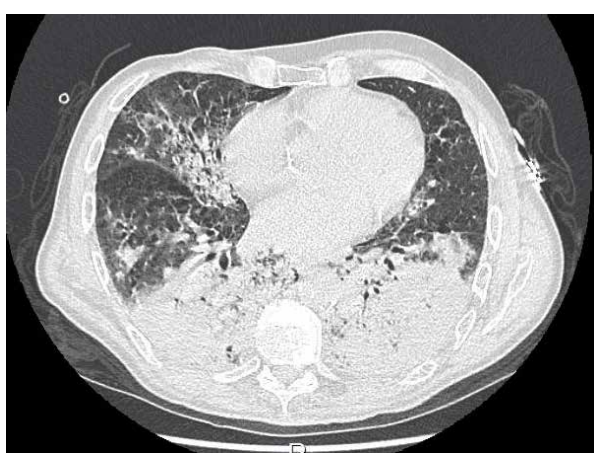

(a)

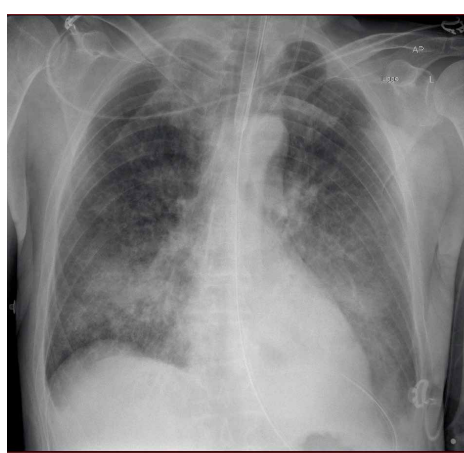

(b)

Figure 1: Computed tomography of lungs at admission (a) and chest X-ray immediately after intubation (b) showing massive bilateral pneumonia.

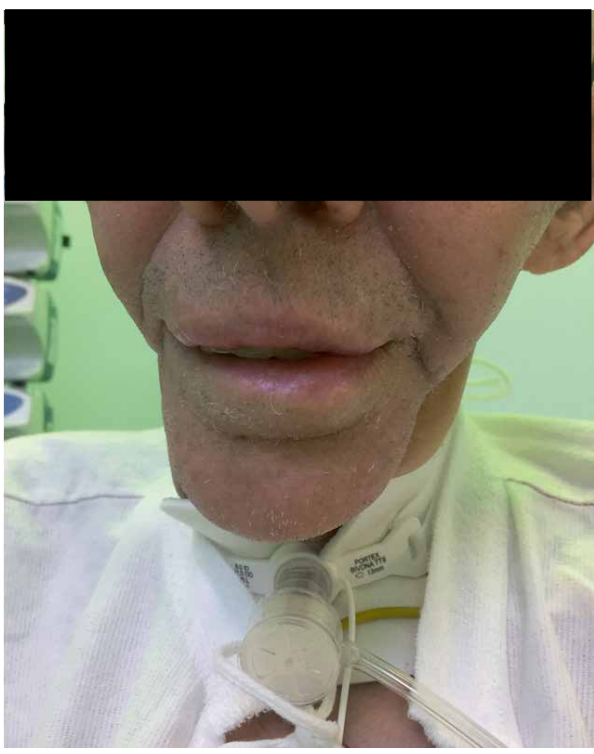

(a)

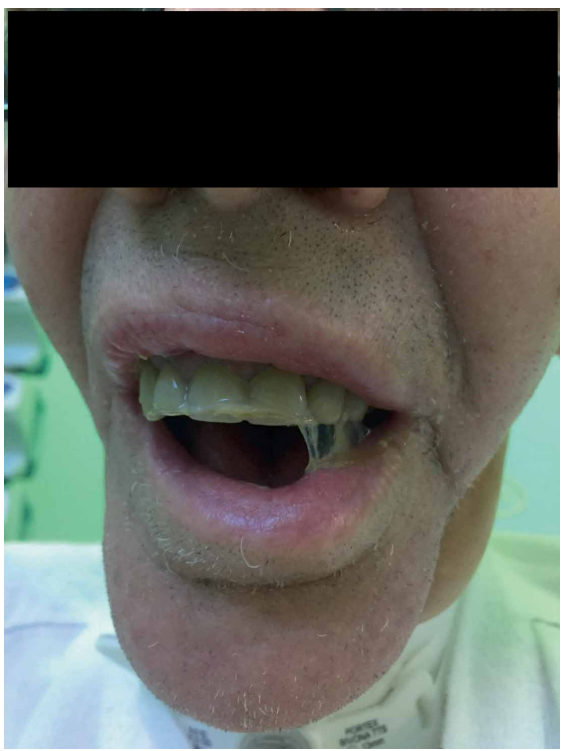

(b)

FIgURE 2: The patient 3 weeks after admission—after surgical tracheostomy ( $\mathrm{a}$ and $\mathrm{b}$ ). Maximal mouth opening of $10 \mathrm{~mm}$ is presented (b).

TABLE 1: Arterial blood gas values at different times before intubation.

\begin{tabular}{lccc}
\hline & $\begin{array}{c}\text { Reservoir-mask } \\
(\mathrm{ER})\end{array}$ & $\begin{array}{c}\text { HFNO } \\
(\mathrm{ICU})\end{array}$ & $\begin{array}{c}\text { NIV } \\
(\mathrm{ICU})\end{array}$ \\
\hline $\mathrm{pH}(7.35-7.45)$ & 7.29 & 7.33 & 7.34 \\
$\begin{array}{l}\mathrm{Base} \text { excess } \\
(-3-3 \mathrm{mmol} / \mathrm{L})\end{array}$ & -10.0 & -8.1 & -8.3 \\
$\mathrm{pO}_{2}(11.1-14.4 \mathrm{kPa})$ & 8.6 & 5.8 & 7.8 \\
Saturation $(\%)$ & 88 & 74 & 88 \\
$\mathrm{pCO}_{2}(4.7-6.4 \mathrm{kPa})$ & 4.3 & 4.3 & 4.0 \\
Lactate & 5.7 & 6.1 & 5.9 \\
$(0.5-1.6 \mathrm{mmol} / \mathrm{L})$ & & & \\
\hline
\end{tabular}

Abbreviations: ER, emergency room; HFNO, high-flow nasal oxygen; ICU, intensive care unit; NIV, noninvasive ventilation.

quickly amended to noninvasive ventilation (NIV) with a positive end expiratory pressure of $12 \mathrm{~cm} \mathrm{H}_{2} \mathrm{O}$ and a $\mathrm{FiO}_{2}$ of $100 \%$. Oxygenation remained unacceptably low (Table 1 ). Accordingly, the decision to intubate was made.
In 1999, the patient's tonsil cancer had been treated with radiation therapy. Unfortunately, this caused mandibular osteoradionecrosis, which had necessitated hemi-mandibulectomy and comprehensive reconstruction surgery (free fibula flap combined with a pedicled pectoralis major myocutaneous flap) on the right side in 2003 and on the left side in 2015. The surgeries were performed following awake FOI and surgical tracheostomy. In 2014, at another hospital, a laparoscopic cholecystectomy necessitated intubation, which was attempted after the induction of anesthesia. A nasal approach utilizing a video laryngoscope (VL) with a hyper angulated blade (McGrath $^{\mathrm{mw}}$ MAC X-Blade ${ }^{\mathrm{mm}}$, Medtronic, MN) failed, and the esophagus was intubated twice. This was attributed to the extreme limited mouth opening, which made visualization of the relevant structures impossible. Further nasal attempts were stopped due to bleeding, and intubation finally succeeded orally with a fiberscope. Since this episode, only awake FOI attempts had been performed.

Other comorbidities included arterial hypertension (treated with an Angiotensin-II-receptor-blocker), chronic jaw 
TABLE 2: Some data on predicted difficulties with intubation and backup plans for oxygenation. Highlighted (with a “+”), which of these features our patient presented $[3,6,7]$.

\begin{tabular}{|c|c|c|c|}
\hline \multirow{2}{*}{$\begin{array}{l}\text { Predictors of difficult intubation } \\
\text { Predictors of difficult direct laryngoscopy }\end{array}$} & \multicolumn{3}{|c|}{ Predictors of difficult backup plans for oxygenation } \\
\hline & \multicolumn{3}{|c|}{ Predictors of difficult facemask ventilation } \\
\hline History of difficult intubation & + & History of neck radiation & + \\
\hline Limited mouth opening (interincisor gap) & + & History of snoring or obstructive sleep apnea & + \\
\hline Modified Mallampati Score $3+4$ & + & Obesity & \\
\hline Limited cervical spine mobility & + & Older age & + \\
\hline Upper lip bite test & + & Male sex & + \\
\hline Limited mandibular protrusion & + & Full beard & \\
\hline Retrognathia & + & Lack of teeth & + \\
\hline \multirow[t]{3}{*}{ Short thyromental distance } & \multirow[t]{3}{*}{+} & Modified Mallampati Score $3+4$ & + \\
\hline & & Limited mandibular protrusion & + \\
\hline & & Short thyromental distance & + \\
\hline Predictors of difficult video laryngoscopy & & Predictors of difficult supraglottic device use & \\
\hline Neck pathology (e.g., scars, neck radiation, mass, thick neck) & + & $\begin{array}{c}\text { Glottic, supraglottic or subglottic pathology (e.g., neck } \\
\text { radiation) }\end{array}$ & + \\
\hline Cormack-Lehane Grade $3+4$ with Macintosh & + & Obesity & \\
\hline Limited cervical spine mobility & + & Older age & + \\
\hline Limited mandibular protrusion & + & Male sex & + \\
\hline \multirow[t]{6}{*}{ Short thyromental distance } & \multirow[t]{6}{*}{+} & Poor dentition & + \\
\hline & & Limited mouth opening & + \\
\hline & & Limited cervical spine mobility & + \\
\hline & & Short thyromental distance & + \\
\hline & & Rotation of surgical table during case & \\
\hline & & Applied cricoid pressure & \\
\hline Predictors of difficult ligthwand use & & Predictors of difficult cricothyrotomy & \\
\hline Obesity (thick neck) & & $\begin{array}{c}\text { Neck surface pathology (e.g. scars, radiation, inflammation, } \\
\text { hematoma) }\end{array}$ & + \\
\hline Limited cervical spine mobility & + & Deviated airway (e.g. goiter, neoplasms) & \\
\hline \multirow[t]{4}{*}{ Large tongue/epiglottis } & \multirow[t]{4}{*}{+} & Obesity (thick neck) & \\
\hline & & Age $<8-10$ years & \\
\hline & & Female sex & \\
\hline & & Limited cervical spine mobility & + \\
\hline
\end{tabular}

pain (treated with oral morphine $40 \mathrm{mg} /$ day) and a history of smoking for 28 years ( $1 / 2$ pack of pipe tobacco/week). Despite his comorbidities, he was physically active and played golf on a weekly basis.

His airway examination revealed a mouth opening of $10 \mathrm{~mm}$ and neck movement $<80^{\circ}$ (due to the bilateral fibrous myocutaneous flaps), among other factors (Figure 2 and Table 2). Most important, however, was the history of previous neck radiation and difficult intubation.

In the ICU, the patient was placed in the upright sitting position to provide airway patency and to ease his respiratory efforts. Glycopyrrolate $0.2 \mathrm{mg}$ IV (Meda, Solna, Sweden) was administered once. Sedation was titrated slowly with midazolam $0.5 \mathrm{mg}$ IV (Hameln, Hameln, Germany) and S-ketamine $2.5 \mathrm{mg}$ IV (Pfizer, Ixelles, Belgium), ensuring slight sedation but cooperation and responsiveness corresponding to a level 2 on the Ramsey Sedation Scale. Airway topicalization was initiated with lidocaine 2\% (Mylan, Canonsburg, PA) $4 \mathrm{~mL}$ nebulized using the NIV machine. This was done twice at 5-minute intervals (total of $160 \mathrm{mg}$ ). Lidocaine 10\% (AstraZeneca,
Södetälje, Sweden) was sprayed onto the base of the tongue and posterior pharynx, subsequently deeper on each of 3 times at 3-4-minute intervals (total of $90 \mathrm{mg}$ ). Transtracheal injection of lidocaine $4 \% 1 \mathrm{~mL}$ was also administered (total of $40 \mathrm{mg}$ ). As potential backup plans, the right nasal cavity was prepared with lidocaine $2 \%$ with epinephrine $5 \mu \mathrm{g} / \mathrm{mL}$, using both a mucosal atomizing device (MAD Nasal ${ }^{\mathrm{m}}$, Teleflex, Wayne, PA) and lidocaine soaked ribbon gauze (total of $20+60 \mathrm{mg}$ ), and infiltration in front of the cricothyroid membrane with $3 \mathrm{~mL}$ (total of $60 \mathrm{mg}$ ) was performed. After 12 minutes of preparation, the patient was changed back from NIV to HFNO and an uneventful oral FOI was performed with a reinforced LMA Fastrach $^{\mathrm{TM}}$ (Teleflex, Waine, PA) endotracheal tube with an internal diameter of $7.5 \mathrm{~mm}$ using the aScope ${ }^{\mathrm{Ts}}$ Regular (Ambu, Ballerup, Denmark). The FOI in itself took less than $2 \mathrm{~min}$. Correct tube placement was confirmed visually and with capnography, Propofol-infusion was initiated $(70 \mu \mathrm{g} / \mathrm{kg} / \mathrm{min})$ and the patient was connected to the ventilator. Five weeks of ventilator therapy followed. Eight days after admission, a surgical tracheostomy was performed at the ENT facility of another 
hospital. The patient was discharged to his home 8 weeks after admission.

\section{Discussion}

National guidelines on the management of difficult airways have been published in numerous countries including the USA, the United Kingdom and Canada [3]. The ultimate goal of all airway management is to avoid a CI-CO scenario, since emergency cricothyrotomy performed by anesthesiologists fails in more than $50 \%$ of these situations $[2,5]$.

For the unexpected difficult airway focus should be on prediction and planning, as to decrease the incidence of its occurrence in the anesthetized and paralyzed patient [3]. Although several bedside tests can be used to anticipate the likelihood of a difficult airway, unfortunately no clinical finding can reliably exclude a difficult airway [6,7]. If difficulties arise after the induction of anesthesia, rescue plans and algorithms should focus on oxygenation and not just center around intubation [3]. Emphasis should be on both technical and non-technical skills [3].

For the expected difficult airway an awake airway securing technique may be the safest option, since it facilitates airway patency, oxygenation and protection against aspiration $[3,4]$. Awake intubation is considered a safe option when difficulties with intubation are anticipated and backup plans for maintaining oxygenation are expected to be difficult (facemask ventilation, supraglottic device (SGD) placement and/or emergency cricothyrotomy) [3, 4]. The presence of obstructing airway pathology, risk of rapid desaturation (e.g. respiratory failure) and increased risk of aspiration all favor an awake approach $[3,8]$.

The first awake FOI was described by Murphy in 1967 [9], and since the 1970's awake FOI has been the "gold standard" for managing anticipated difficult airways $[3,4,10]$. In recent years, awake VL intubation has been described with similar success rates as awake FOI [10]. In cases of uniquely altered anatomy (e.g., limited mouth opening, HNC in the oral cavity or upper airway and previous radiation), insertion of the VL blade with free sight may, however, be impossible [11]. In our case, use of a VL had previously been impossible due to the extremely limited mouth opening. Usually, the following minimal mouth opening must be present for reliable airway maneuvers: Macintosh intubation $>25 \mathrm{~mm}$, SGD usage $>25 \mathrm{~mm}$ and VL intubation $>20 \mathrm{~mm}[8,12,13]$. Nevertheless, successful use of these devices in patients with less mouth opening has been reported [14].

Experts disagree as to whether awake FOI skills should be mastered by every anesthesiologist or only restricted to practitioners who deal with these difficult airways on a daily basis [15]. A survey among US anesthesiologists in 2003 found that only $59 \%$ reported to have skills in FOI [16]. Opportunities to acquire FOI skills in the operating room have decreased since the introduction of the VL in clinical practice in 2002. An acceptable level of technical skills may be acquired after performing 10 FOI's in general anesthesia and 15-20 on awake patients $[8,17]$, but true expertise probably requires more training. This necessitates heavy weighting of trainees performing supervised FOI's, optimally as part of a routine institutional practice [4]. New airway simulators consisting of components including replica video bronchoscopes, and desktop sensors can create virtual patients with difficult airways. This possibly may accelerate obtaining FOI skills, even though transferability of these skills to performance in real life patients with difficult airways has not yet been shown.

In a recent review on elective awake FOI protocols in the operating room, no specific strategy regarding premedication, local anesthesia, or sedation could be declared superior to the others [5]. In the critically ill patient, with minimal physiological reserves, airway obstruction (due to over-sedation) and respiratory or circulatory collapse would be dangerous. Sedation should thus be kept to a minimum (or no sedation at all) and nonopioid medication including ketamine may be the safest choice. In the end, familiarity of the anesthesiologist performing the FOI with their drug of choice is probably the most important point [5].

The disadvantage of FOI is that blood and secretions in the airway or at the tip of the fiberscope may obscure the vision. A thin suctioning catheter can be placed nasally in the pharynx for continuous suctioning and cautious low-flow oxygen through the working channel of the fiberscope can be administered to continuously flush the tip. Both of these techniques were prepared for, but not needed since visibility was obtained immediately. Some argue that FOI is extremely time consuming. However, with the right preparations undertaken simultaneously, this may not be true $[8,11]$.

Other theoretical options would have been classical blind techniques used for decades, such as blind nasal [18], retrograde [19] or light wand intubation [20]. Nevertheless, these are increasingly becoming a lost art and even if local expertise exists, success rates are not necessarily acceptable [21]. It is possible that an oral retromolar approach with a rigid intubating stylet (e.g., Bonfils ${ }^{\mathrm{TM}}[22]$ ) or a nasal approach with the newer rigid video-intubating stylet (Trachway $^{\mathrm{TM}}$ [23]) may have worked, but equipment and expertise are not available in all departments. Placing the patient on extracorporeal membrane oxygenation (ECMO) before performing a surgical tracheostomy has been reported in extreme circumstances [24]. However, besides potential risk of major hemorrhaging, highly specialized equipment and expertise in thoracic and ENT surgery are required.

The airway plan was an awake oral FOI. It was preferable to avoid the nasal route, since it was probably necessary to decrease the tube size and since nasal bleeding would have been disastrous. Accordingly, an awake nasal FOI was the second choice. As backup plan in hospitals with ENT surgical expertise an awake surgical tracheostomy would have been a viable option or could have represented the primary choice instead of the awake FOI. In hospitals without ENT surgical expertise (as in this case), an awake percutaneous dilatational tracheostomy (if experienced intensivists are present) or an awake cricothyrotomy could have been the potential backup plan, keeping in mind that these procedures may be technically very challenging, when performed in local anesthesia in the critically ill patient on spontaneous breathing. The last option in either case would have been an emergency cricothyrotomy performed under adequate anesthesia and full relaxation. 
Once the transcricothyroidal local anesthetic was given, using an adequately sized plastic cannula instead of the smallbore needle and leaving it on site as a backup for cricothyrotomy would have been a possibility, providing access for temporary emergency jet oxygenation or for placement of a guidewire in the trachea in connection with Seldinger based cricothyrotomy sets.

\section{Conclusion}

This case illustrates that patients with difficult airways may present at hospitals without ENT expertise, requiring urgent intubation, which is not manageable with conventional methods as well as VL. Optimal skills for performing an awake FOI should be present around the clock. As backup, an awake tracheal access can be considered: awake surgical tracheostomy (if ENT surgical expertise is present), awake percutaneous dilatational tracheostomy (if specially experienced intensivists are present) or awake cricothyrotomy (if neither ENT surgeons or intensivists are present). These methods may be the only options to avoid emergency cricothyrotomy, which has an unacceptable high failure rate when performed by an anesthesiologist in a CI-CO scenario.

\section{Conflicts of Interest}

The authors declare that they have no conflicts of interest.

\section{References}

[1] http://esundhed.dk/sundhedsregistre/CAR/CAR03/Sider/ Tabel.aspx.

[2] C. V. Rosenstock, A. K. Nørskov, J. Wetterslev, and L. H. Lundstrøm, "Emergency surgical airway management in Denmark: a cohort study of 452461 patients registered in the Danish Anaesthesia Database," British Journal of Anaesthesia, vol. 117, pp. i75-i82, 2016.

[3] J. A. Law, N. Broemling, R. M. Cooper et al., "The difficult airway with recommendations for management: Part 2: The anticipated difficult airway," Canadian Journal of Anesthesia, vol. 60, no. 11, pp. 1119-1138, 2013.

[4] L. Cabrini, M. B. Redaelli, L. Ball et al., "Awake fiberoptic intubation protocols in the operating room for anticipated difficult airway: a systematic review and meta-analysis of randomized controlled trials," Anesthesia and Analgesia, vol. 128, no. 5, pp. 971-980, 2019.

[5] T. M. Cook, N. Woodall, and C. Frerk, "Major complications of airway management in the UK: results of the Fourth National Audit Project of the Royal College of Anaesthetists and the Difficult Airway Society. Part 1: anaesthesia," British Journal of Anaesthesia, vol. 106, no. 5, pp. 617-631, 2011.

[6] T. Shiga, Z. Wajima, T. Inoue, and A. Sakamoto, "Predicting difficult intubation in apparently normal patients," Anesthesiology, vol. 103, pp. 429-437, 2005.

[7] M. E. Detsky, N. Jivraj, N. K. Adhikari et al., "Will this patient be difficult to intubate? The rational clinical examination systematic review," JAMA, vol. 321, no. 5, pp. 493-503, 2019.
[8] K. E. Hannig, C. Jessen, R. W. Hauritz, and A. M. Grejs, "Awake fiberoptic intubation in fast track ambulatory surgery: a case report," A\&A Practice, vol. 11, no. 6, pp. 165-168, 2018.

[9] P. Murphy, "A fibre-optic endoscope used for nasal intubation," Anaesthesia, vol. 22, no. 3, pp. 489-491, 1967.

[10] M. Alhomary, E. Ramadan, E. Curran, and S. R. Walsh, "Videolaryngoscopy vs. fibreoptic bronchoscopy for awake tracheal intubation: a systematic review and meta-analysis," Anaesthesia, vol. 73, no. 9, pp. 1151-1161, 2018.

[11] T. T. Joseph, J. S. Gal, S. DeMaria, Hung-Mo Lin, Adam I. Levine, and Jaime B. Hyman, "A retrospective study of success, failure, and time needed to perform awake intubation," Anesthesiology, vol. 125, no. 1, pp. 105-114, 2016.

[12] G. Aiello and I. Metcalf, "Anaesthetic implications of temporomandibular joint disease," Canadian Journal of Anaesthesia, vol. 39, no. 6, pp. 610-616, 1992.

[13] M. Lawrence, D. Ball, A. Braga, G. Hotvedt, and G. Rodney, "Trismus and the limits of laryngoscopy," Anaesthesia, vol. 69, no. 12, pp. 1401-1402, 2014.

[14] J. R. Maltby, R. G. Loken, M. T. Beriault, and D. P. Archer, "Laryngeal mask airway with mouth opening less than $20 \mathrm{~mm}$," Canadian Journal of Anaesthesia, vol. 42, no. 12, pp. 1140-1142, 1995.

[15] I. Ahmad and C. R. Bailey, "Abandoning awake fibreoptic bronchoscopy - a reply," Anaesthesia, vol. 71, no. 5, pp. 595-596, 2016.

[16] T. Ezri, P. Szmuk, R. D. Warters, J. Katz, and C. A. Hagberg, "Difficult airway management practice patterns among anesthesiologists practicing in the United States: have we made any progress?", Journal of Clinical Anesthesia, vol. 15, no. 6, pp. 418-422, 2003.

[17] T. Heidegger, H. J. Gerig, B. Ulrich, and T. W. Schnider, "Structure and process quality illustrated by fibreoptic intubation: analysis of 1612 cases," Anaesthesia, vol. 58, no. 8, pp. 734-739, 2003.

[18] I. W. Magill, "Endo-tracheal anaesthesia," Proceedings of the Royal Society of Medicine, vol. 22, pp. 83-88, 1928.

[19] F. S. Butler and A. A. Cirillo, "Retrograde tracheal intubation," Anesthesia and Analgesia, vol. 39, no. 4, pp. 333-338, 1960.

[20] R. Macintosh and H. Richards, "Illuminated introducer for endotracheal tubes," Anaesthesia, vol. 12, no. 2, pp. 223-225, 1957.

[21] M. F. Aziz, A. M. Brambrink, D. W. Healy et al., "Success of intubation rescue techniques after failed direct laryngoscopy in adults," Anesthesiology, vol. 125, no. 4, pp. 656-666, 2016.

[22] S. Y. Thong and G.-L. T. Wong, "Clinical uses of the Bonfils retromolar intubation fiberscope: a review," Anesthesia and Analgesia, vol. 115, no. 4, pp. 855-866, 2012.

[23] M. C. Lee, K. Y. Tseng, Y. C. Shen et al., "Nasotracheal intubation in patients with limited mouth opening: a comparison between fibreoptic intubation and the Trachway "' Anaesthesia, vol. 71, no. 1, pp. 31-38, 2016.

[24] G. Malpas, O. Hung, A. Gilchrist et al., "The use of extracorporeal membrane oxygenation in the anticipated difficult airway: a case report and systematic review," Canadian Journal of Anesthesia, vol. 65, no. 6, pp. 685-697, 2018. 


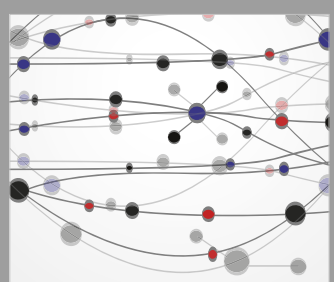

The Scientific World Journal
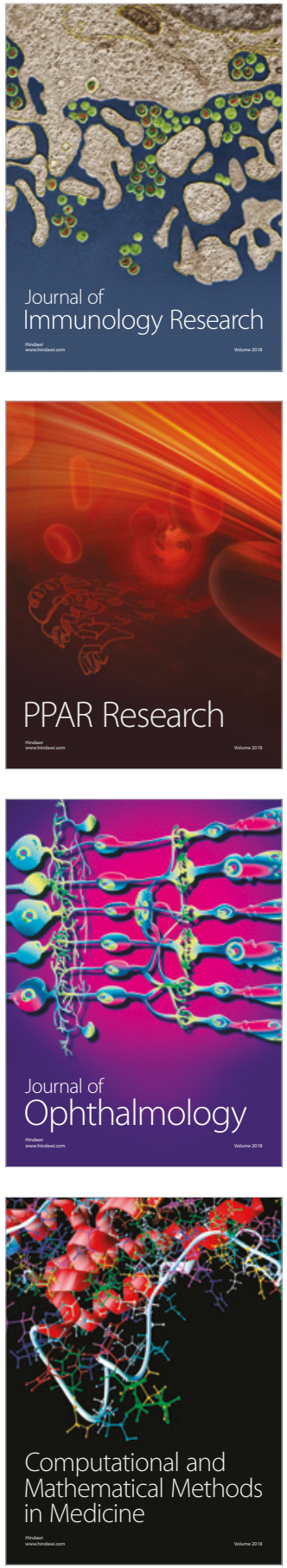

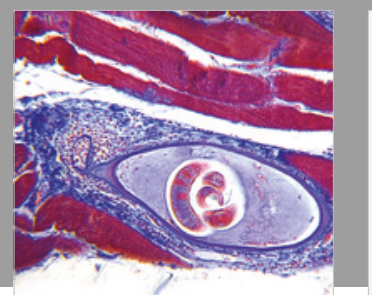

Gastroenterology Research and Practice

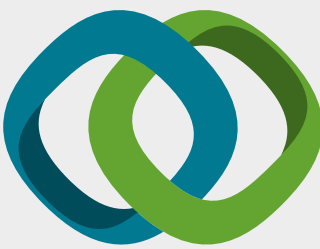

\section{Hindawi}

Submit your manuscripts at

www.hindawi.com
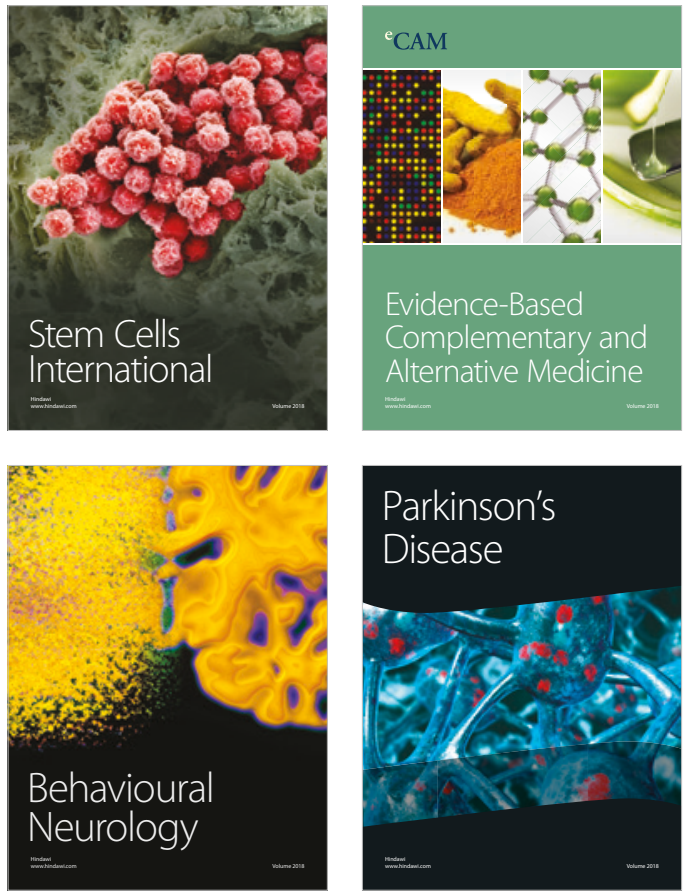

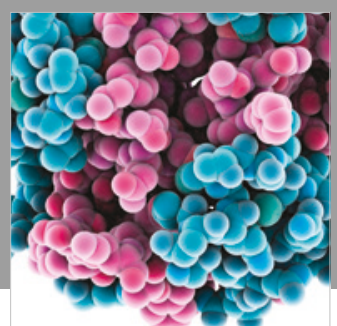

ournal of

Diabetes Research

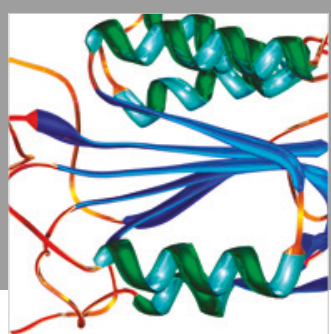

Disease Markers
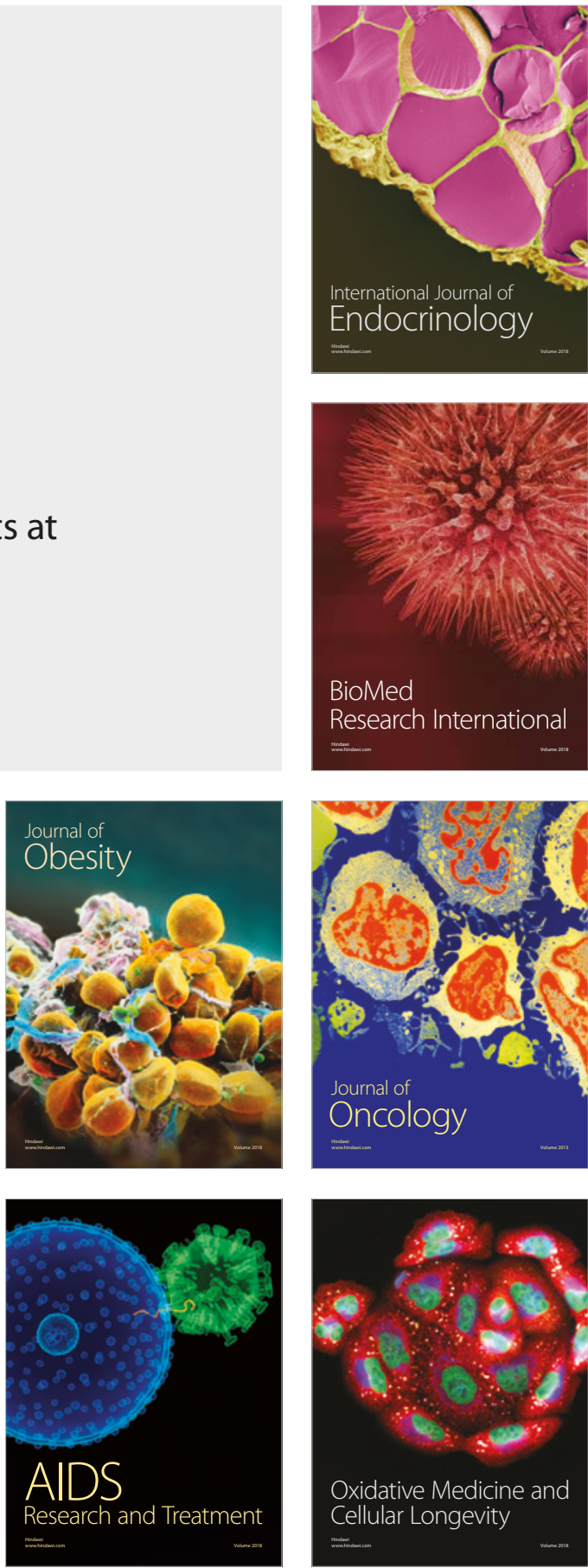